\title{
1 A shale rock physics model for analysis of brittleness index, 2 mineralogy, and porosity in the Barnett Shale
}

\author{
Zhiqi Guo ${ }^{1}$, Xiang-Yang $\mathrm{Li}^{2,3}$, Cai Liu' ${ }^{1}$, Xuan Feng ${ }^{1}$, and Ye Shen ${ }^{4}$ \\ ${ }^{1}$ Jilin University, No.938 Xi Minzhu Street, Changchun city, China, 130021; \\ ${ }^{2}$ British Geological Survey, West Mains Road, Edinburgh, Great Britain, EH9 3LA; \\ ${ }^{3}$ China University of Petroleum, No.18 Fuxue Street, Changping Qu, Beijing city, China, \\ 102249. \\ ${ }^{4}$ CNOOC Research Institute, No.6, Dongzhimenwai Xiaojie, Beijing, China \\ Email: guozhiqi@yahoo.com.cn
}

\begin{abstract}
We construct a rock physics workflow to link elastic properties of shales to complex constituents and specific microstructure attributes. The key feature in our rock physics model is the degrees of preferred orientation of clay and kerogen particles defined by the proportions of such particles in their total content. The self-consistent approximation (SCA) method and Backus averaging method are used to consider the isotropic distribution and preferred orientation of compositions and pores in shales. Using the core and well log data from the Barnett Shale, we demonstrate the application of the constructed templates for the evaluation of porosity, lithology, and brittleness index. Then, we investigate the brittleness index defined in terms of mineralogy and geomechanical properties. The results show that as clay content increases, Poisson's ratio tends to increase, and Young's modulus tends to decrease. Moreover, we find that Poisson's ratio is more sensitive to the variation in the texture of shales resulting from the preferred orientation of clay particles. Finally, based on the constructed rock physics model, we calculate AVO responses from the top and bottom of the Barnett Shale, and the results indicate predictable trends for the variations in porosity, lithology, and brittleness index in shales.
\end{abstract}

Keywords: shale rock physics, brittleness index, mineralogy, porosity 


\section{Introduction}

As a unique type of lithology, shales compose a major component of sedimentary rocks. Because more unconventional resources are being explored in shale sequences than ever before, a better understanding on the relationship between elastic properties and microstructures in shales tends to be increasingly important. Shales represent anisotropy, and the transverse isotropic of shales is regarded as the results from the preferred orientation of clay particles, which can be related to the depositional and stress history (Sayers 1994, 2005; Slatt and Abousleiman 2011). At the same time, petrophysical analyses of petroleum source rocks (Vernik and Nur 1992; Vernik and Landis 1996) indicate that strong velocity anisotropy can also be associated with the presence of organic matter and its distribution in the rock matrix, depending on the richness and maturity of organic matter.

Due to high degree of heterogeneity and anisotropic features, linking complex microstructure and composition to elastic anisotropy in shales tends to be a challenging task. For the modeling of shale anisotropy, two categories of effective medium theories are commonly used: the Backus averaging method (Vernik and Nur 1992; Carcione 2000) and the anisotropic self-consistent approximation (SCA) method (Hornby 1994; Sayers 1994, 2005; Johansen, Jakobsen, and Ruud 2002; Levin and Markov 2005).

If shales have relatively simple mineralogical compositions and laminated structures, the Backus averaging method works well in the modeling of shale elastic anisotropy. On the other hand, if shales are composed of complicated mineralogical constituents and represent complex microstructures, the anisotropic SCA method for heterogeneous media can give more accurate estimate of shale elastic anisotropy than the Backus averaging method. However, the sophisticated anisotropic SCA methods require more complicated input parameters including shapes and orientation distributions of mineral particles and cracks. These parameters are obtained from the statistical analysis of advanced imaging techniques (e.g., scanning electron microscopy [SEM] micrographs), but in practice the accurate quantification of these parameters is not always easy to conduct.

The purpose of our study is to find a compromise for the modeling of elasticity of structural and layered shales, considering both heterogeneity and laminated structures in shales. In our heuristic model, the key parameters are the degrees of preferred orientation of clay and/or kerogen particles, which are defined by the proportions of such particles in their total content. First, we build a workflow for the heuristic shale rock physics model based on the effective medium theories. Then, we generate rock physics templates to illustrate the effect of lithology and porosity on seismic attributes and geomechanical properties of shales, and analyze the shale brittleness index defined 
in terms of mineralogy and geomechanical properties in the rock physics templates. Also, we investigate how the degree of preferred orientation of clay particles affect seismic attributes, geomechanical, and brittleness index. We validate the shale rock physics model on the well log data from the Barnett Shale, and use the rock physics template for the evaluation of the brittleness index of the Barnett Shale. Finally, based on the predicted elastic properties on the templates, we model the AVO responses from the top and the bottom of the Barnett Shale and investigate if there are predictable trends for the variations in porosity, lithology, and brittleness index on the AVO intercept-gradient crossplot.

\section{Shale rock physics model}

The key factor in our model is the degree of preferred orientation of clay particles and/or kerogen. Total contents of clay minerals and kerogen are considered as two portions: one consists of particles of disordered distribution and the other consists of particles of preferred orientation. The scenarios of clay particles and/or kerogen with three different degrees of preferred orientation are illustrated in the schematic in figure 1(a). The panel in figure 1(b) illustrates the schematic of workflow for our shale rock physics model. Firstly, the proportions of isotropic distributed minerals, kerogen, and pores/cracks are modeled using effective medium theory to obtain isotropic effective elastic properties. Secondly, elastic anisotropy resulting from the proportion of preferred orientations of clay particles and/or kerogen is calculated using the Backus averaging method. In this stage, the modeled shales represent transverse isotropy with vertical symmetric axis. Finally, for the possible presence of vertical fractures, the resulted orthorhombic anisotropy can be modeled using existing effective medium theories. The detection for the vertical fractures can be conducted using cross-dipole measurements or can be inferred from various imaging logging techniques.

Figure 2 illustrates the associated effective medium theories in each stage of the shale rock physics workflow. In the first step, clay with micropores of bound water is modeled using the self-consistent approximation (SCA) method. The mixture of kerogen and pores saturated with oil, gas, and water is modeled using the approach given by Carcione (2010), in which wood's equation and the model of Kuster and Toksöz (1974) were used to obtain the properties of the mixture. In the second step, the elastic properties of the proportion consisting of randomly distributed particles and fluid-saturated pores and cracks are modeled using the isotropic SCA method proposed by Berryman (1980, 1995). In the next step, the VTI elastic anisotropy of shales resulting from the preferred orientation of clay particles and/or kerogen are obtained using the Backus averaging method by incorporating the outputs from the previous step. Finally, elastic anisotropy resulting from possible presence of vertical fractures can be modeled by existing 
method such as Hudson's effective medium theories (Hudson, 1980). The details of the SCA and the Backus averaging method used in this paper are illustrated as the followings.

\subsection{Self-consistent approximation (SCA) model}

The self-consistent effective media approximation method proposed by Norris et al (1985) and Kazatchenko et al (2004) was applied by Aquino-López (2011) to conduct joint simulation of elastic moduli and electrical conductivity for clean sand formations. In this study, however, we focus on the generalized n-phase SCA method developed by Berryman $(1980,1995)$ to link complex constituents and fluid-saturated pores with different geometries to the elastic properties of shales. This method provides the estimate of self-consistent elastic modulus $K_{S C}^{*}$ and $\mu_{S C}^{*}$ of rocks given n phases of mineralogy and pore-space:

$$
\sum_{j=1}^{n} f_{j}\left(K_{j}-K_{S C}^{*}\right) \beta^{* j}=0
$$

$$
\sum_{j=1}^{n} f_{j}\left(\mu_{j}-\mu_{S C}^{*}\right) \zeta^{* j}=0
$$

where, each $j$ indicates a phase of mineralogy or pore space with a corresponding volume fraction $f_{j}$ and bulk $\left(K_{j}\right)$ and shear $\left(\mu_{j}\right)$ modulus for that phase. We consider prolate ellipsoidal inclusions with aspect ratio $\alpha \leq 1$, so the factors $\beta^{* j}$ and $\zeta^{* j}$ that describe the geometry of an inclusion made of phase $j$ within a background medium (denoted with subscript $\mathrm{m}$ ) are given by

$$
\beta^{* j}=\frac{K_{m}+\frac{4}{3} \mu_{j}}{K_{j}+\frac{4}{3} \mu_{j}+\pi \alpha \mu_{m} \frac{3 K_{m}+\mu_{m}}{3 K_{m}+4 \mu_{m}}}
$$

and

As an inclusion model, the SCA isolates the pores from one another, preventing hydraulic communication and pore-pressure equilibrium. Thus, Equations 1 to 4 describe a high-frequency model and are often used for ultrasonic laboratory data where the high frequencies do not allow enough time for pore pressures to equilibrate. As to the low-frequency application, Mavko et al (2009) recommend a SCA-Gassmann method, which the SCA method is used for a dry rock frame, followed by Gassmann theory for fluid substitution. In this work, because of low porosity 
and very low permeability in shales, the assumption of the SCA as a high-frequency model appears to be satisfied. Thus, we treat pores and cracks saturated with mixtures of gas, oil, and water as inclusions in the SCA method.

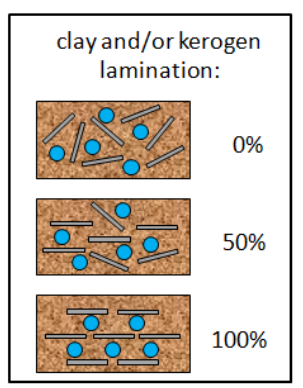

(a)

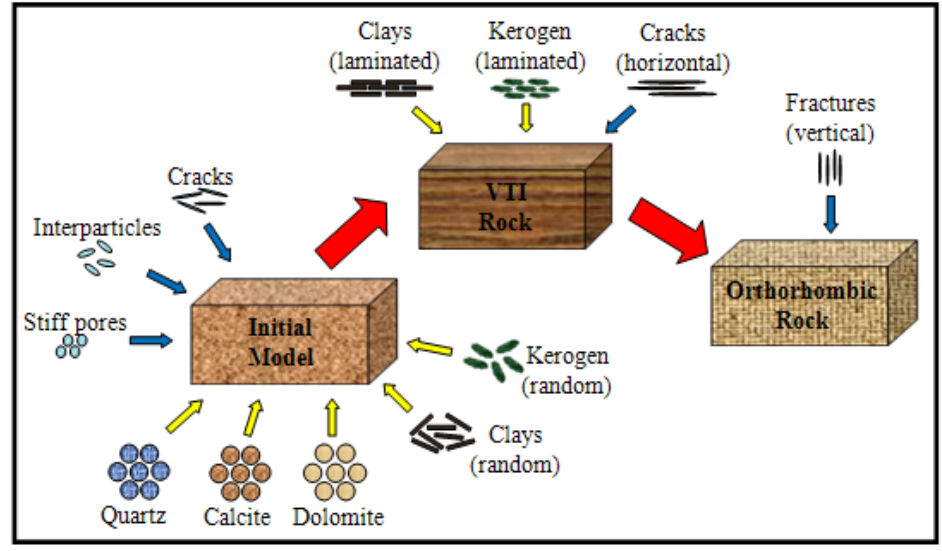

(b)

Figure 1 Diagrams illustrate the schematic of different clay lamination (left) and the workflow of the shale rock physics model (right).

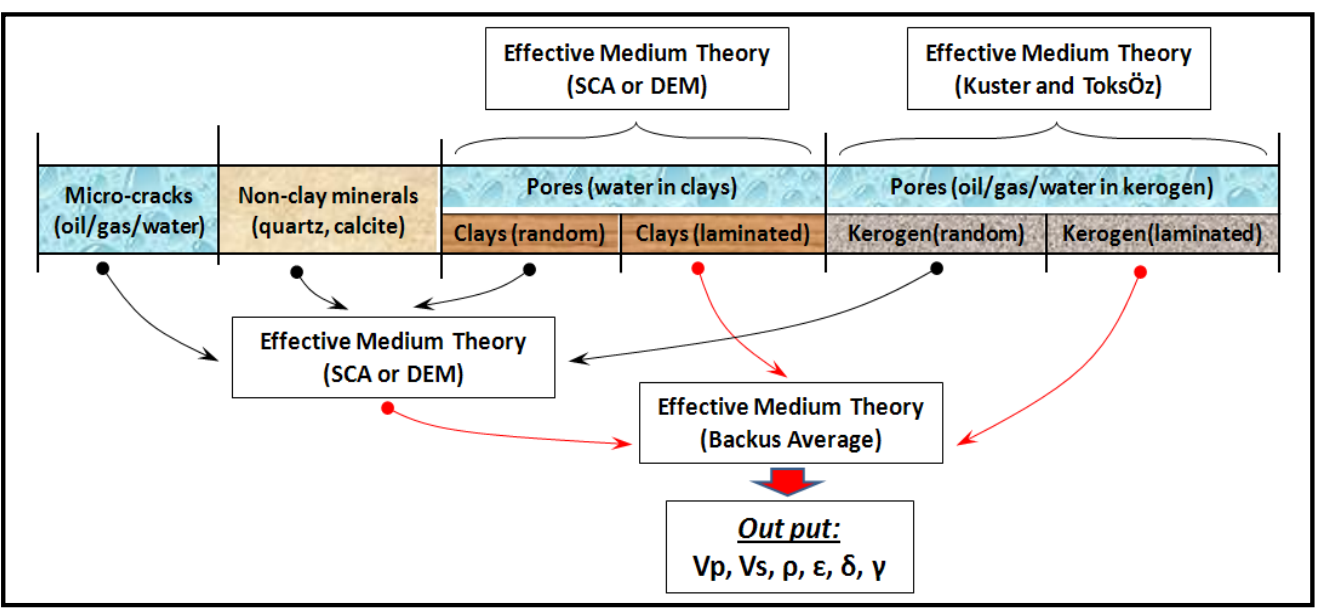

Figure 2 Diagram shows the effective medium theories used in the shale rock physics model.

Depending on the microstructures of rocks, a single- or multiple-aspect-ratio model is usually used for the modeling of pore space (Xu and Payne 2009; Ruiz and Chen 2010). In this work, we investigate a multiple-aspect-ratio model, and incorporate statistical distribution of pore geometries for a more realistic representation of the pore space (Spikes 2011; Jiang and Spikes 2011). The aspect ratio of each set of pore inclusions has a normal distribution, specified by a mean value and a standard deviation. The standard deviation is set to be a tenth of the mean value, and each set of pores consists of a number of 100 aspect ratios (Guo et al 2012).

\subsection{Anisotropic Backus averaging method}

In the work of Vernik and Nur (1992), they assumed that the shale rock is a multiple-layer composite made of laminated clay minerals and kerogen of lamination in texture, and the mixture 
of all other minerals. Anisotropic Backus averaging gives a transversely isotropic equivalent medium described by five effective stiffnesses,

$$
\begin{gathered}
c_{11}^{*}=\left\langle c_{11}-c_{13}^{2} c_{33}^{-1}\right\rangle+\left\langle c_{33}^{-1}\right\rangle^{-1}\left\langle c_{33}^{-1} c_{13}\right\rangle^{2}, \\
c_{33}^{*}=\left\langle c_{33}^{-1}\right\rangle^{-1}, \\
c_{13}^{*}=\left\langle c_{33}^{-1}\right\rangle^{-1}\left\langle c_{33}^{-1} c_{13}\right\rangle, \\
c_{55}^{*}=\left\langle c_{55}^{-1}\right\rangle^{-1}, \\
c_{66}^{*}=\left\langle c_{66}\right\rangle .
\end{gathered}
$$

In equation (5), the weighted average of a physical quantity $\alpha$ according to our rock physics model is defined as

$$
\langle\alpha\rangle=f_{c} \alpha_{c}+f_{k} \alpha_{k}+f_{m} \alpha_{m}
$$

where the subscripts $c, k$, and $m$ indicate laminated clay mineral and kerogen, and the mixture all other compositions, respectively. Parameter $f$ denotes weight for averaging.

\section{Rock physics templates}

Rock physics templates are used to visualize lithological and mineralogical variations in terms of derived seismic attributes, and can be applied for the quantitative interpretation of well log and seismic data. Perez et al (2011) constructed rock physics templates using a combination of Hertz-Mindlin contact theory and the lower modified Hashin-Shtrikman bounds to guide interpretations of estimated ultimate recovery (EUR) in shales. The model they used was under isotropic assumption and considered simple mineralogical mixture of quartz-clay. In this work, we investigate the effect of complex mineralogical constituents, and the variations in porosity and texture in shales based on our anisotropy shale rock physics model.

\subsection{Seismic attributes related to mineralogy and porosity in shales}

We apply the shale rock physics model to construct rock physics templates and display them in figure 3. The results represent the modeled elastic properties in the vertical direction. In the modeling, mineralogy of shales is composed of clay, dolomite, quartz, and kerogen, and the mineralogical substitution occurs between clay and dolomite. We model the scenario that when clay content increases from $10 \%$ to $40 \%$ with an interval of $10 \%$, dolomite content decreases from $40 \%$ to $10 \%$. We keep the content of quartz and kerogen constant, at $40 \%$ and $10 \%$, respectively, and assume that kerogen has a disordered orientation, and clay particles have a degree of preferred orientation of $50 \%$. Kerogen-related porosity is set to be $20 \%$, and the aspect ratio of 
fluid-saturated pores is set to be 0.1. Material properties of associated minerals in the modeling are given in table 1.

Rock physics templates illustrate the mapping of lines of constant porosity and mineralogical mixtures on the crossplots of elastic seismic attributes and geomechanical properties. Solid lines indicate the constant values of clay content, and dashed lines represent constant values of porosity. The intersection points of these lines are overlaid with dots, representing selected values of porosity, from 0 to 0.20 with an increment of 0.04 . Specific colours of dots indicate constant clay content, explained by the legend in figure 3.

Figure 3(a) shows the crossplot of $V p / V s$ and $I p$. We can see that the variation in porosity for each lithology is ready to be distinguished from the attributes of both $V p / V s$ and $I p$. On the other hand, we find for each constant porosity line that the discrimination of the clay content using $V p / V s$ represents similar resolution, but the same work using Ip appears to be more difficult as porosity increases. For porosity higher than 0.16 , there is no evident variation in Ip for different lithology.

\subsection{Brittleness index of shales}

There are two common ways to define rock brittleness index. One way is to calculate the brittleness index in terms of the proportion of brittle minerals (e.g., quartz, dolomite) in shales. The presence of such minerals makes shales more brittle while the presence of more clay mineral makes shales more ductile. The work of Miller et al (2007) indicated that an increased quartz-clay ratio correlates to lower breakdown pressures for stimulation and enhance relative production. The other way to define the brittleness index is in terms of geomechanical properties of Young's modulus $E$ and Poisson's ratio $v$. Shales with higher $E$ and lower $v$ tend to be more brittle (Rickman et al 2008). Waters et al (2011) gave a definition of the Brittleness Index (BI),

$$
B I=\frac{\left[\frac{100\left(E-E_{\min }\right)}{\left(E_{\max }-E_{\min }\right)}+\frac{100\left(v-v_{\max }\right)}{\left(v_{\min }-v_{\max }\right)}\right]}{2},
$$

where $E$ and $v$ are Young's modulus and Poisson's ratio at each depth location along well path. $E_{\min }$ and $E_{\max }$ are minimum and maximum vertical Young's modulus in interval of interest; $v_{\min }$ and $v_{\max }$ are minimum and maximum vertical Poisson's ratio in interval of interest, respectively.

On the other hand, Goodway et al (2007) related $E$ and $v$ to the more seismically intuitive Lame parameters of incompressibility $\lambda$ and rigidity $\mu$ through the following relationships

$$
E=\frac{\mu(3 \lambda+2 \mu)}{\lambda+\mu},
$$




$$
v=\frac{\lambda}{2(\lambda+\mu)},
$$

and found that the increase in $\mu$ leads to the increase in $E$ and the decrease in $v$. Therefore, $\quad \mu$ may represent a good indicator of $B I$.

Figure 3(b) illustrates the template in terms of the geomechanical properties of $E$ and $v$. From each constant porosity lines, we can see that the increase in clay content will decrease the values of $v$, but $E$ tends to be less sensitive to the variation in clay content as porosity increases. This may imply that compared to $E, v$ is a more reliable parameter for the discrimination of geomechanical brittleness of shales.

Figure 3(c) shows the $\lambda \rho-\mu \rho$ crossplot introduced by Goodway et al (2007) for improved fluid detection and lithology discrimination. The parameter of $\lambda \rho$ and $\mu \rho$ are measures of incompressibility and rigidity of rocks, respectively. We can see that $\mu \rho$ decreases as clay content increases for various porosities, but $\lambda \rho$ may represents opposite trends. This proves $\lambda \rho$ to be a good indicator for lithology and brittleness index.

\subsection{Mineralogical substitution: a different scenario}

We investigate another mineralogical substitution between clay and quartz mineral and show the resulted templates in figure 4, displaying the same attributes as those in figure 3. In this case, when clay content increases from $10 \%$ to $40 \%$ with an interval of $10 \%$, quartz content decreases from $40 \%$ to $10 \%$. We keep dolomite content as a constant of $40 \%$ and set other parameters the same as those in the case shown figure 3 .

Similarly, the attributes of $V p / V s$ and $v$ show their ability for the discrimination of lithology and shale brittleness index. Compared to the dolomite substitution, the major difference in this case is a wider range of the variations in the attributes of $V p / V s, v, \lambda \rho$. Such difference may be explained by the difference in elastic properties between the minerals of quartz and dolomite.

\subsection{Clay lamination}

As illustrated in figure 1, the key parameter in our shale rock physics model is the degree of preferred orientation of clay particles. In figure 5 and figure 6 , we examine how this factor affects geomechanical properties of $E$ and $v$. From figure 5(a), (b) and (c), we can see that the increasing degree of clay lamination has very subtle influence on $E$ but significant impact on $v$. In the three templates in figure 5, for the same lithology and porosity denoted by the colour-coded dots, the increase in the degree of preferred orientation of clay particles increase the value of $v$, which mean that the presence of more laminated textures of clay particles make shales more ductile. 
Figure 6 shows the effect of the variation in clay lamination for the case of the mineralogical substitution between clay and quartz. Similar trends as those in figure 5 can be found in the templates as the degree of clay lamination increases.

\section{Calibration on well log data}

We apply our shale rock physics model to the Barnett Shale to predict its elastic properties along with the variations in porosity and mineralogy. Figure 7 shows the well logs of the Barnett Shale surrounded by the overlying Marble Falls formation and the underlying Ellenburger formation. The Barnett Shale is readily distinguishable in the higher values of gamma ray and lower values of $V_{P}, V_{S}$, and $\rho$. Red dots along depth in the gamma ray panel indicate the locations where core samples are taken. Figure 8(a) illustrates the measured mineralogical volumetric fraction and figure 8(b) the corresponding ternary plot of compositions from the core data. The ternary plot shows that quartz and clay dominate the mineralogical content in the Barnett Shale. Material properties of minerals and pore fluids used in the modeling are shown in table 1.

Figure 9 shows the templates incorporating the effects of porosity, mineralogy, and brittleness index in the Barnett Shale on the crossplots of seismic attributes $V_{P} / V_{S}$ and $I_{P}$. The panel in figure 9(a) is colour-coded by $B I$ defined by geomechanical properties and the panel in figure 9(b) is colour-coded by the content of brittle mineralogy. In the modeling, the mineralogical substitution occurs between clay and quartz. While clay content increases from 0 to $60 \%$, quartz content reduces from $60 \%$ to 0 . We assume the aspect ratio of pores in shales has a value of 0.1 according to the study of Guo et al (2012), and kerogen has a disordered orientation. Qualitatively, the degree of preferred orientation of clay particles of $20 \%$ fits the superimposed data points in the modeling and interprets the well-log-derived seismic attributes with a reasonable accuracy. As shown on the templates, porosity has a value less than $12 \%$, and clay content has a spread from zero to around $40 \%$ with dominant values between $20 \%$ and $40 \%$, which are consistent with those measured from well log data in figure 7 and core data in figure 8.

The absolute values of the two brittleness indices are different because they are based on two different definition system. By comparison, we find that the variations in the two definitions of $B I$ according to porosity and clay content are consistent for clay content higher than about $25 \%$. As clay content increases, the content of brittle mineralogy decreases, and the geomechanical $B I$ decreases accordingly. This difference may result from the fact that geomechanical properties of shales are the complex function of both mineralogy and microstructure, rather than mineralogy along. In this study, we demonstrate the results of $B I$ defined in terms of two common ways, geomechanical properties and mineralogy, on the rock physics templates, but do not tend to discuss which one is more applicable than the other in practice. 
Thus, the constructed rock physics templates can be used to quantitatively predict and interpret well log and seismic data for the estimation of porosity and mineralogy and the evaluation of brittleness index.

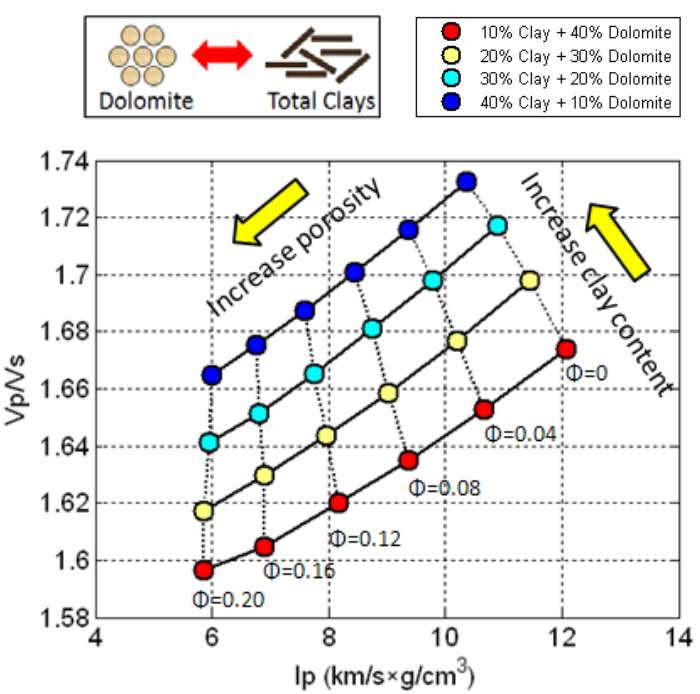

(a)

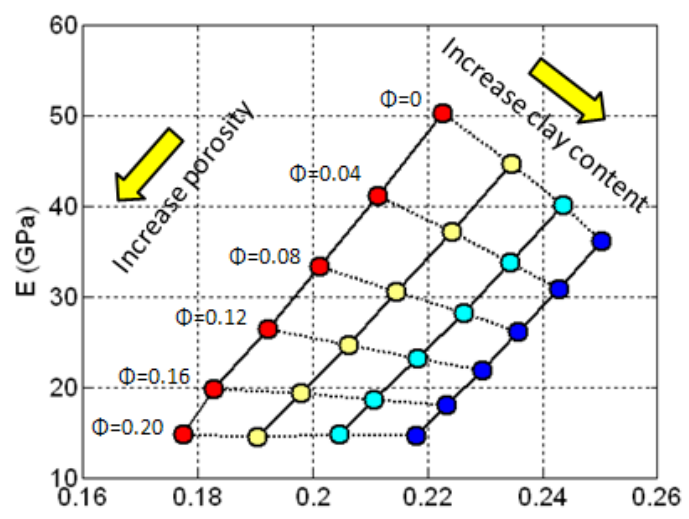

(b)

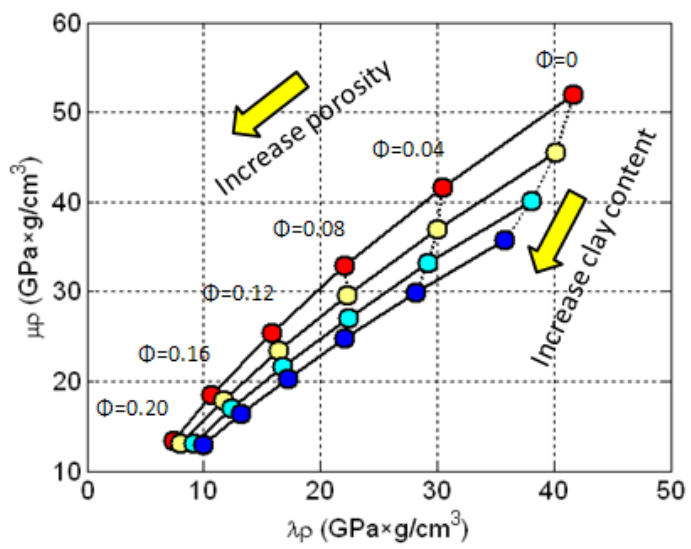

(c)

Figure 3 Rock physics templates showing the effect of mineralogical mixtures and porosity on the crossplots of (a) $I p$ versus $V p / V s$, (b) $v$ versus $E$, and (c) $\lambda \rho$ versus $\mu \rho$. Mineralogical substitution occurs between dolomite and clay.
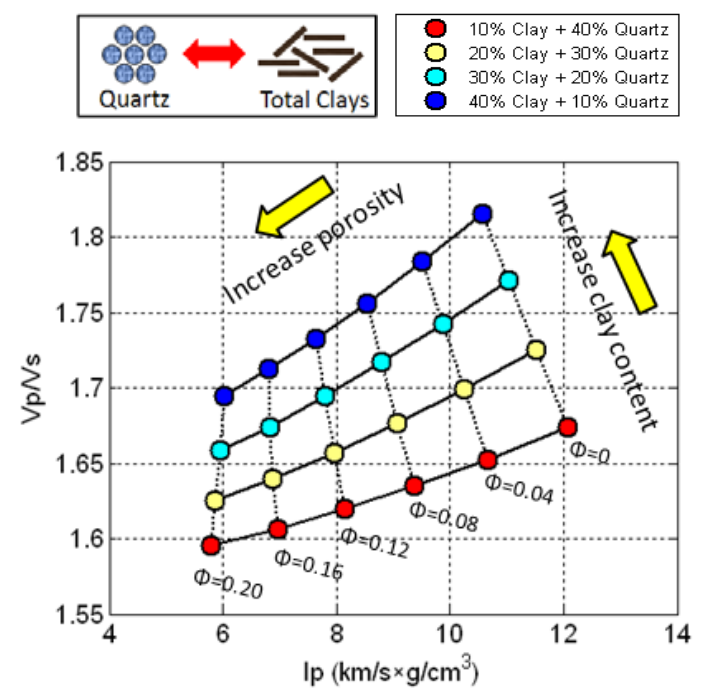

(a)

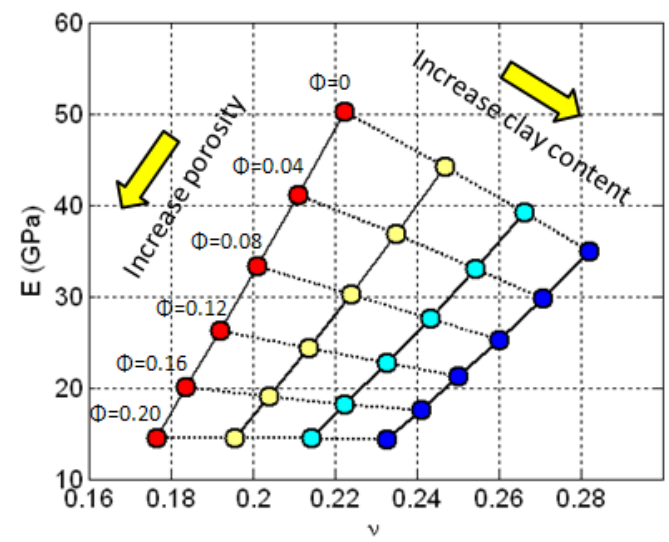

(b)

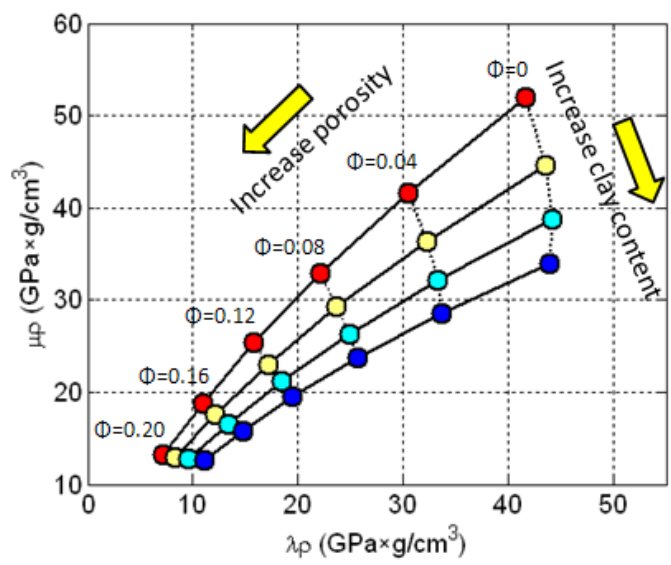

(c)

Figure 4 Rock physics templates showing the effect of mineralogical mixtures and porosity on the crossplots of (a) $I p$ versus $V p / V s$, (b) $v$ versus $E$, and (c) $\lambda \rho$ versus $\mu \rho$. Mineralogical substitution occurs between quartz and clay. 

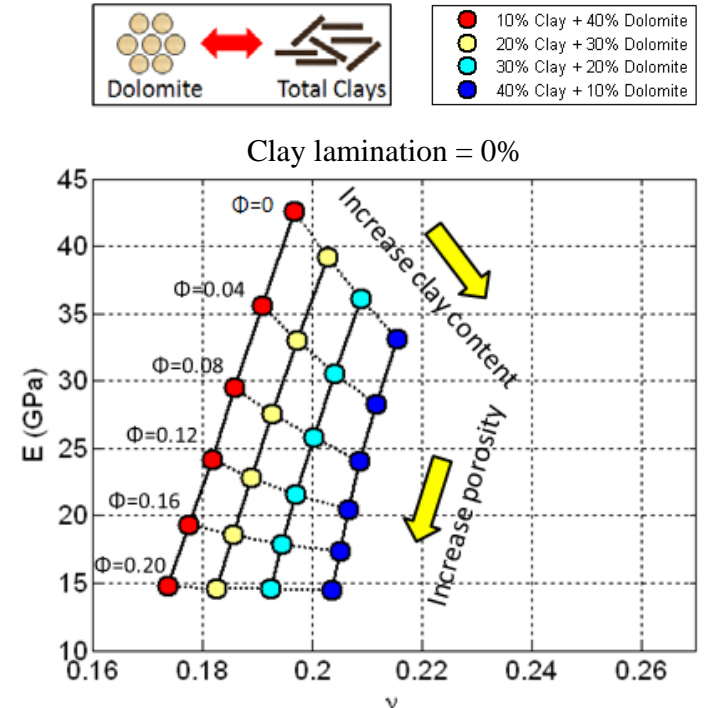

(a)

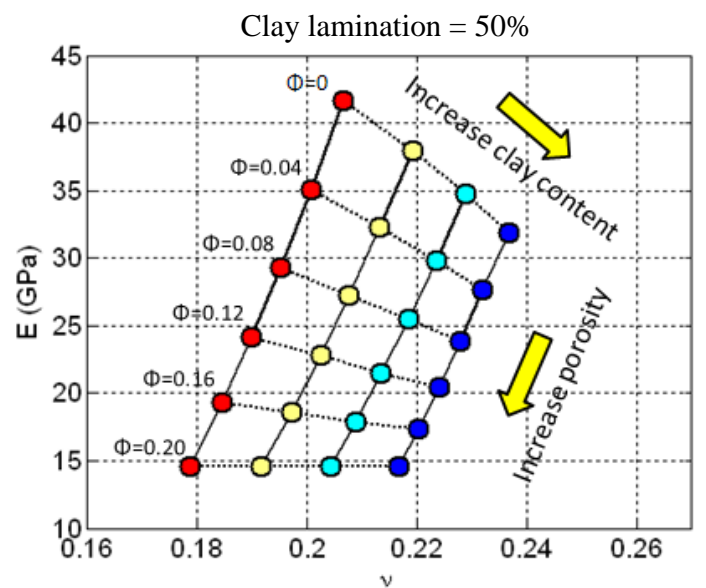

(b)

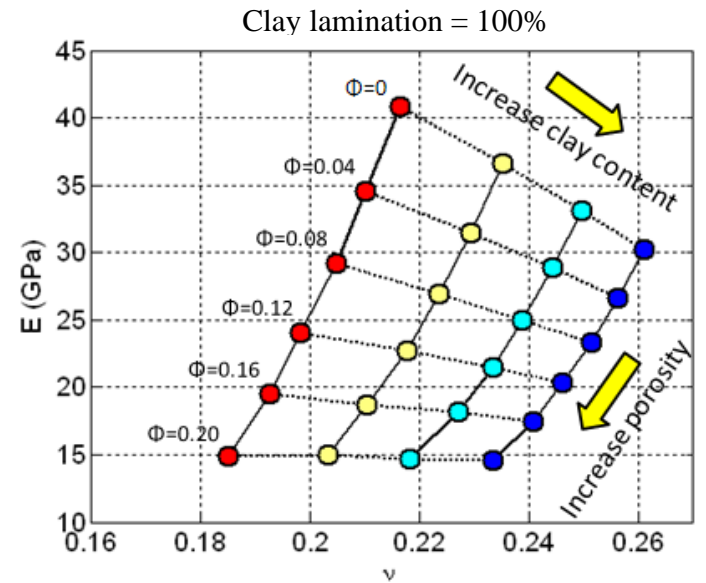

(c)

Figure 5 Crossplots of acoustic impedance $I p$ versus $V p / V s$, showing the degree of clay, (a) $0 \%$, (b) $50 \%$, and (c) $100 \%$. Mineralogical substitution occurs between dolomite and clay.

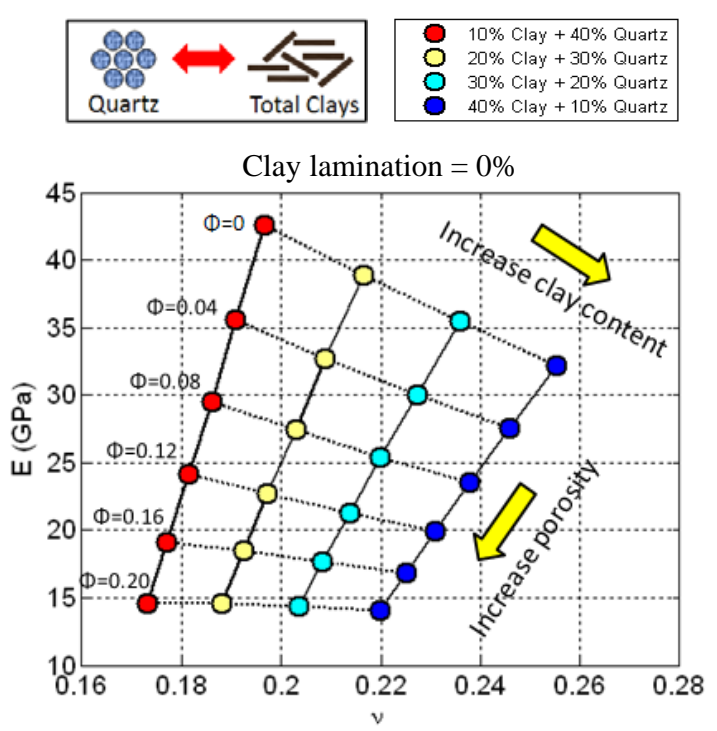

(a)

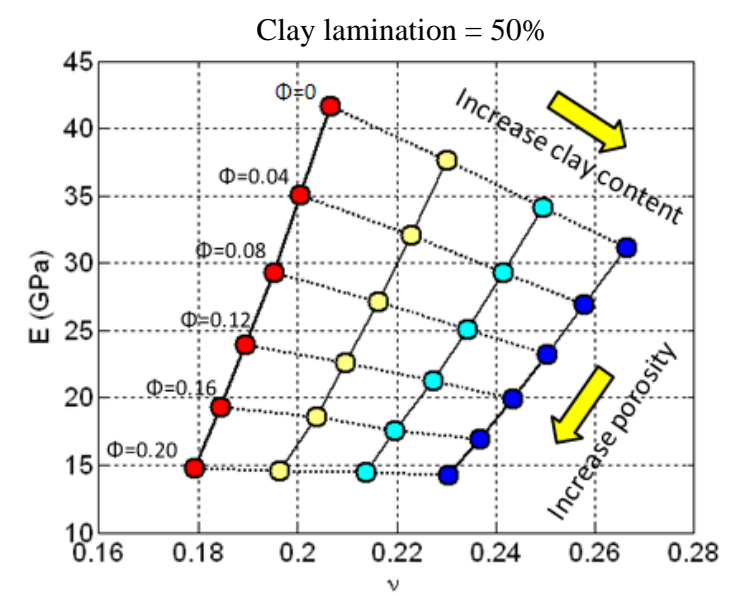

(b)

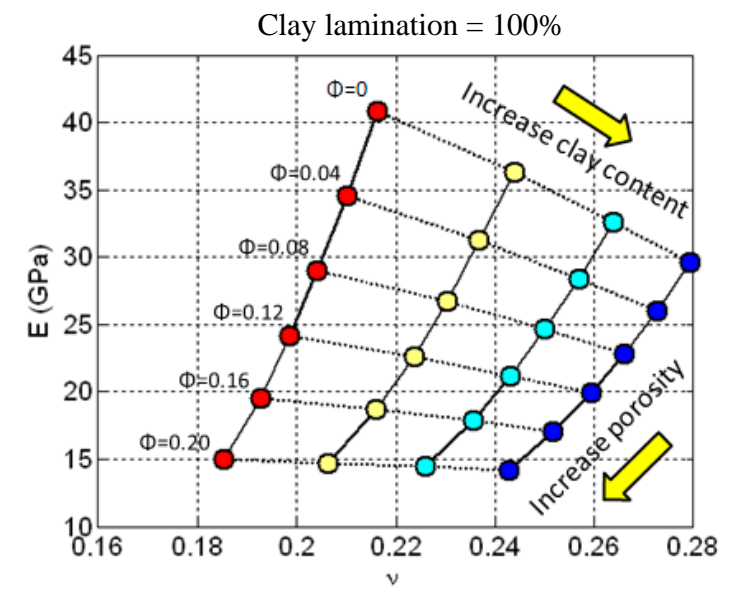

(c)

Figure 6 Crossplots of acoustic impedance $I p$ versus $V p / V s$, showing the degree of clay, (a) $0 \%$, (b) $50 \%$, and (c) $100 \%$. Mineralogical substitution occurs between quartz and clay. 


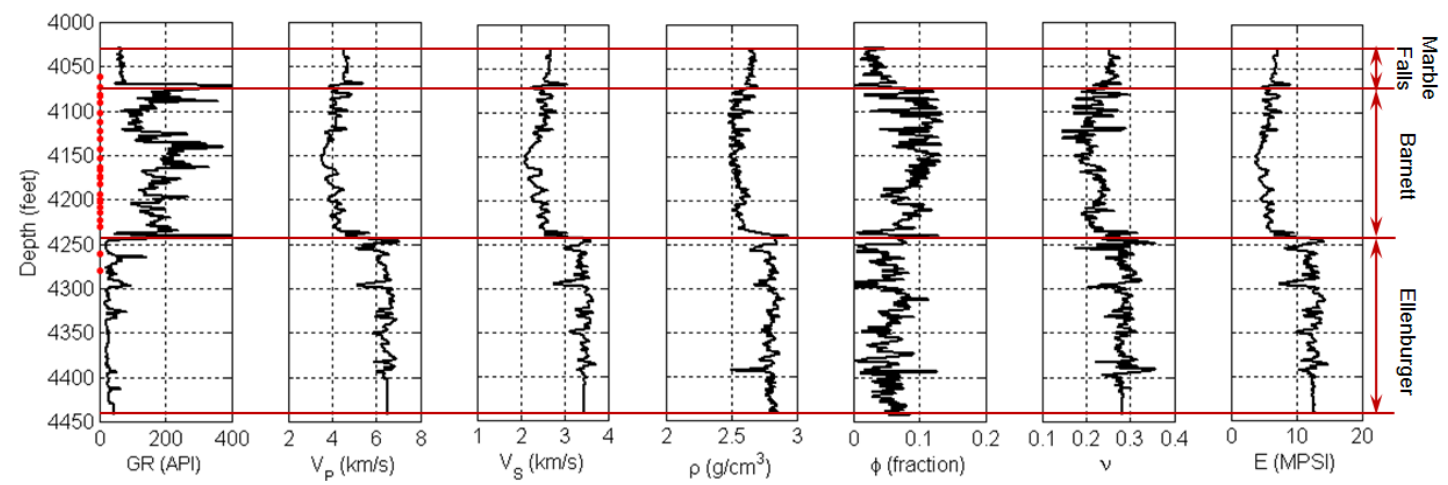

Figure 7 Well data of the three formations: Marble Falls Limestone, Barnett Shale, and the Ellenburger Formations. Well logs are (from left to right) the gamma ray, $\mathrm{P}$-wave velocity $(V p)$, S-wave velocity $(V s)$, density $(\rho)$, porosity $(\varphi)$, Poisson's ratio $(v)$, and Young's modulus $(E)$. The red lines delineate the contacts among the three formations evaluated.

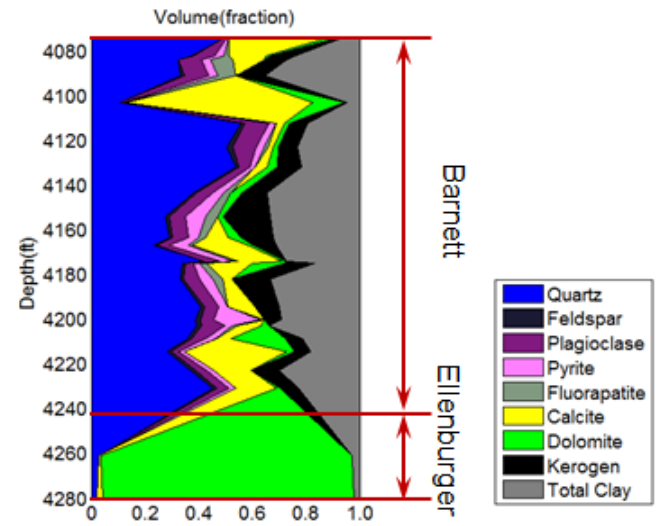

(a)

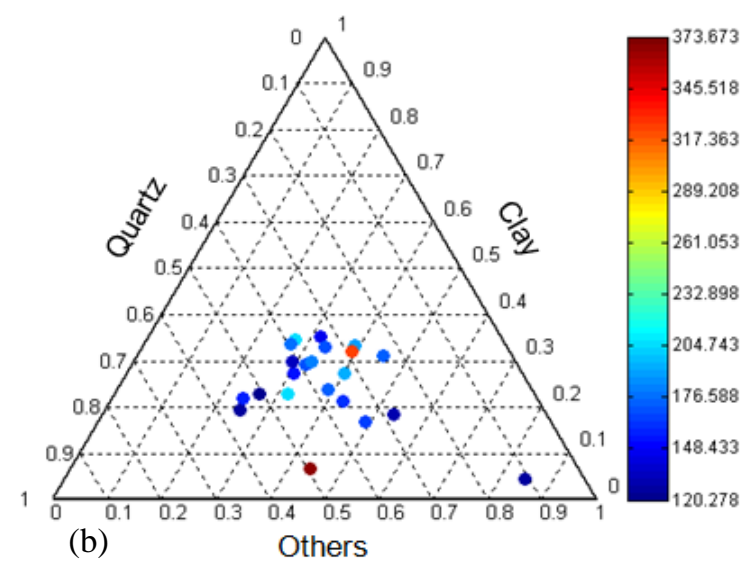

(b)

Figure 8 (a) Mineral volumetric fraction. (b) Ternary plot of compositions colour-coded by gamma ray values.

Table 1 Material properties

(References in Mavko et al 2009)

\begin{tabular}{lccc}
\hline & $\begin{array}{c}\text { Density } \\
\left(\mathrm{kg} / \mathrm{m}^{3}\right)\end{array}$ & $\begin{array}{c}\text { Bulk modulus } \\
(\mathrm{GPa})\end{array}$ & $\begin{array}{c}\text { Shear modulus } \\
(\mathrm{GPa})\end{array}$ \\
\hline Quartz & 2650 & 37 & 44 \\
Feldspar & 2620 & 38 & 15 \\
Plagioclase & 2630 & 76 & 26 \\
Pyrite & 4810 & 147 & 133 \\
Fluorapatite & 3210 & 87 & 47 \\
Calcite & 2710 & 77 & 32 \\
Dolomite & 2870 & 95 & 45 \\
Kerogen & 1300 & 2.9 & 2.7 \\
Clay & 2500 & 25 & 9 \\
Oil & 700 & 0.57 & 0 \\
Gas & 111 & 0.04 & 0 \\
Water & 1040 & 2.25 & 0 \\
\hline
\end{tabular}


As we notice, however, some data points fall out of the modeled zero porosity line, and such data have higher $\mathrm{P}$-wave impedance and higher $\mathrm{Vp} / \mathrm{Vs}$ ratio (or Poisson's ratio). The mis-fit may result from the assumption of constant value of pore aspect ratio, because although the work of Guo et al (2012) indicates that the value of pore aspect ratio is stable and has a mean value around 0.1 in the Barnett Shale, there does exist some local fluctuations in the value of pore aspect ratio which introduce an uncertainty in our rock physics model. In this case, modeling heterogeneous microstructures on the templates is challenging because the variation in pore geometry has a great impact on elastic properties of shales.

\section{Seismic AVO responses study of the Barnett formation}

According to the results in figure 9, elastic properties of the Barnett Shale vary along with porosity and lithology. Such variations have impact on the seismic responses from the top and bottom of the Barnett Shale. We calculate the AVO responses corresponding to the predicted elastic properties in figure 9 and illustrate the derived crossplots of AVO intercept $P$ and gradient $G$ in figure 10. Figure 10 (a) and (b) are also colour-coded by brittleness indices in terms of geomechanical properties and mineralogy, respectively. We can find that the $P$ - $G$ crossplots shows predictable trend in the variations of mineralogical contents, porosity, and brittleness index of the Barnett Shale.

\section{Discussions and conclusions}

We have built a rock physics workflow to model the complexity of constituents and microstructures of shales. The key factor in our rock physics model is the degrees of preferred orientation of clay or kerogen particles defined by the proportions of such particles in their total content. This factor allows us to model depositional and diagenetic features of shales.

Based on the rock physics model, we constructed rock physics templates in terms of seismic attributes and geomechanical properties for the analysis of porosity, lithology, and brittleness index. The results show that seismic attributes of both $V p / V s$ and $I p$ can reflected the variation in porosity, but compared to $I p, V p / V_{s}$ has much sensitivity for the variation in clay content, especially as porosity increases. 
On the rock physics templates, we found that as clay content increases, Poisson's ratio $v$ tends to decrease, and Young's modulus $E$ tends to decrease, which justify the consistency of the brittleness index defined in terms of geomechanical properties and lithology. However, as porosity increases, Poisson's ratio tends to be a more reliable indicator for the discrimination of clay content than Young's modulus. Another important result is that Poisson's ratio is sensitive to the variation in the texture of shales resulting from the preferred orientation of clay particles.
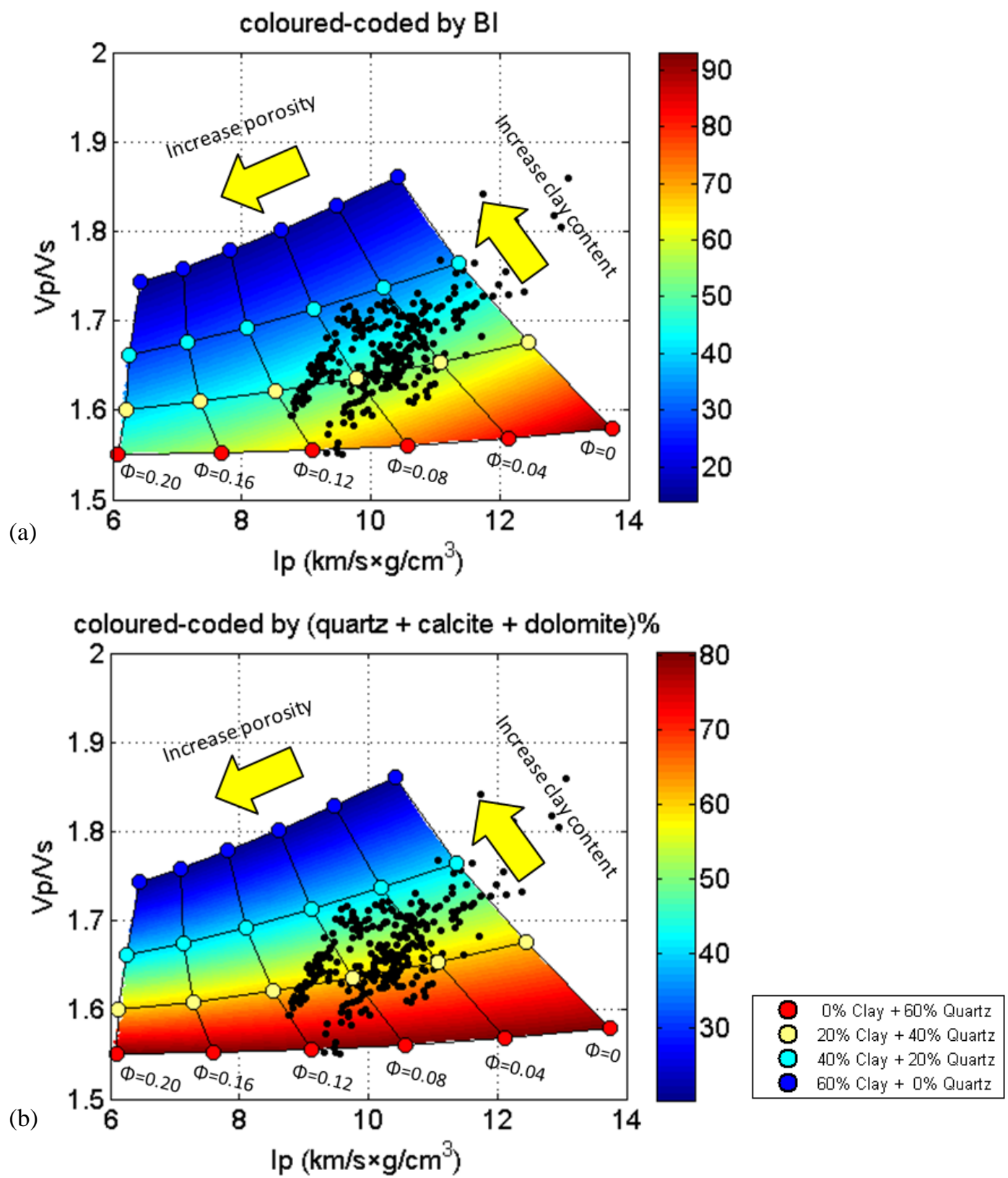

Figure 9 Shale rock physics templates of $I p$ versus $V p / V s$ showing the effect of mineralogy, porosity, and brittleness index. The templates are colour-coded by (a) BI (upper) and (b) the content of brittle mineralogy (lower) respectively to show the brittleness index for the Barnett Shale. 


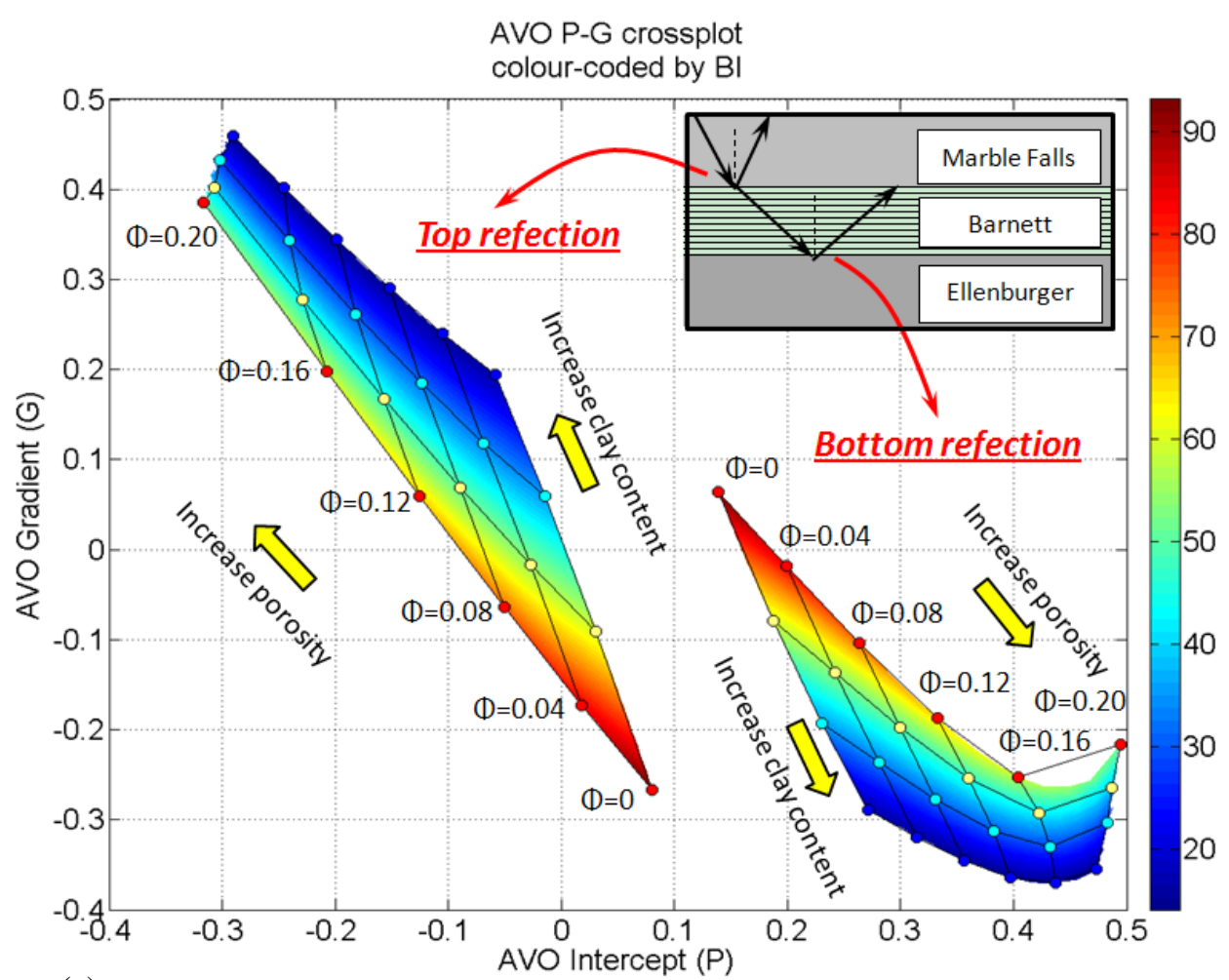

(a)

AVO P-G crossplot

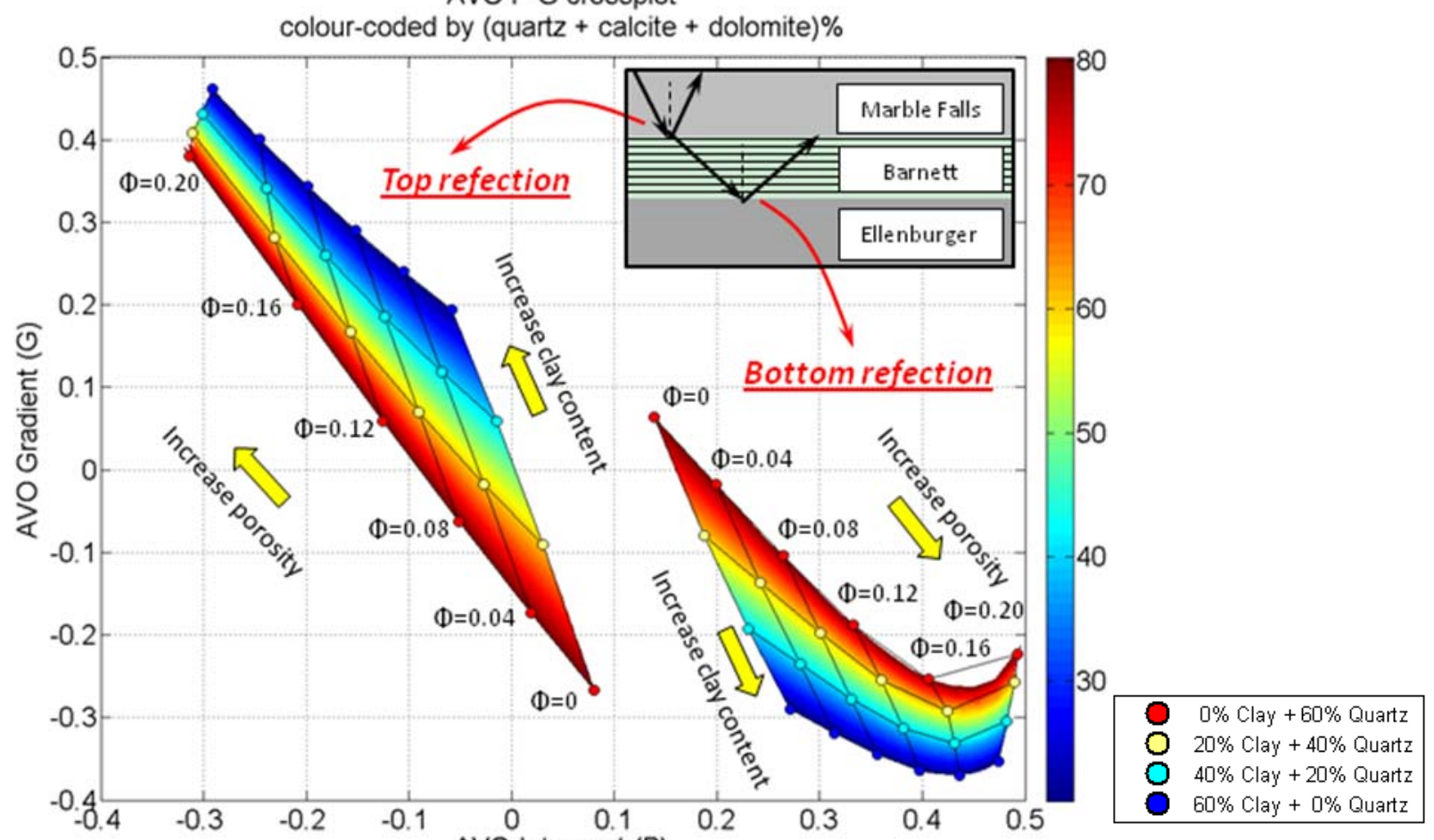

(b)

Figure 10 Crossplots of AVO intercept (P) and gradient (G) for the AVO responses from the top and the bottom of the Barnett Shale. The crossplots are colour-coded by (a) BI (upper) and (b) the content of brittle mineralogy (lower) respectively to show the brittleness index for the Barnett Shale. 
Core and well log data validate the application of our rock physics model for the evaluation of porosity, lithology, and brittleness index. Also, AVO responses of the Barnett Shale represented in terms of intercept-gradient crossplots indicate the predictable trends for the variation in porosity, lithology, and brittleness index.

\section{Acknowledgements}

The work is presented with the permission of the EAP Sponsors and the Executive Director of the British Geological Survey (NERC), and is partially supported by the Research Project of Jilin University (No. 450060481096). We thank all anonymous reviewers for their important comments to improve this paper.

\section{References}

Aquino-López A, Mousatov A and Markov M 2011 Model of sand formations for joint simulating the elastic moduli and electrical conductivity Journal of Geophysics and Engineering 8 568-578

Berryman J G 1980 Long-wavelength propagation in composite elastic media The Journal of the Acoustical Society of America 68 1809-31

Berryman J G 1995 Mixture theories for rock properties, in rock physics and phase relations A Handbook of Physical Constants vol 3 ed T J Ahrens (Washington, DC: AGU) pp 205-28 AGU Ref Shelf

Carcione J 2000 A model for seismic velocity and attenuation in petroleum source rocks Geophysics 65 (4) 1080-92

Goodway B, Varsek J and Abaco C 2007 Isotropic AVO methods to detect fracture prone zones in tight gas resource plays 2007 CSPG CSEG Convention 585-9

Guo Z Q, Li X Y, and Chapman M 2012 Correlation of brittleness index with fractures and microstructure in the Barnett Shale $74^{\text {th }}$ EAGE Conference \& Exhibition, Extended Abstracts F022

Hornby B E, Schwartz L M and Hudson J A 1994 Anisotropic effective medium modeling of the elastic properties of shales Geophysics $\mathbf{5 9} 1570-83$ 
Hudson J A 1980 Overall properties of a cracked solid Math. Proc. Camb. Phil. Soc. 88 $371-84$.

Jiang M and Spikes K T 2011 Pore-shape and composition effects on rock-physics modeling in the Haynesville Shale 81st SEG Meeting Expanded Abstracts 2079-83

Johansen T A, Jakobsen M and Ruud B O 2002 Estimation of the internal structure and anisotropy of shales from borehole data Journal of Seismic Exploration 11 363-381

Kazatchenko E, Markov M and Mousatov A 2004 Joint inversion of acoustic and resistivity data for carbonate microstructure evaluation Petrophysics 45 130-40

Kuster G and ToksÖz M 1974. Velocity and attenuation of seismic waves in two-phase media: Part I - Theoretical formulations Geophysics 39 587-606

Levin V M and Markov M G 2005 Elastic properties of inhomogeneous transversely isotropic rocks Int. J. Solids and Str. 42 393-408.

Mavko G, Mukerji T and Dovrkin J 2009 The Rock Physics Handbook (2 ${ }^{\text {nd }}$ edition) (Cambridge University Press)

Miller C, Lewis R and Bartenhagen K 2007 Design and execution of horizontal wells in gas shales using borehole images and geochemical data Search and Discovery Article \#90065

Norris A N, Sheng P and Callegari A J 1985 Effective-medium theories for two-phase dielectric media J. Appl. Phys. 57 1990-6

Perez M., Close D, Goodway B and Purdue G 2011 Developing templates for integrating quantitative geophysics and hydraulic fracture completions data: part I - principles and theory 81st SEG Meeting Expanded Abstracts 1794-98.

Rickman R, Mullen M, Petre E, Grieser B and Kundert D 2008 A practical use of shale petrophysics for simulation design optimization: All Shale plays are not clones of the Barnett Shale SEP 115258

Ruiz F and Chen A 2010 A rock physics model for tight gas sand The Leading Edge 29(12) $1484-89$

Sayers C M 1994 The elastic anisotropy of shales Journal of Geophysical Research 99 $767-74$

Sayers C M 2005 Seismic anisotropy of shales Geophysical Prospecting 53 667-76. 
Slatt R and Abousleiman Y 2011 Merging sequence stratigraphy and geomechanics for unconventional gas shales The Leading Edge 30(3) 274-82

Spikes K T 2011 Modeling elastic properties and assessing uncertainty of fracture parameters in the Middle Bakken Siltstone Geophysics 76(4) E117-26

Vernik L and Nur A 1992 Ultrasonic velocity and anisotropy of hydrocarbon source rocks Geophysics $\mathbf{5 7}$ 727-35

Vernik L and Landis C 1996 Elastic anisotropy of source rocks: Implication for HC generation and primary migration AAPG Bull $80531-44$

Waters G A, Lewis R E and Bentley D C 2011 The effect of mechanical properties anisotropy in the generation of hydraulic fractures in organic shales SPE 146776

Xu S and Payne M A 2009 Modeling elastic properties in carbonate rocks The Leading Edge 28(1) 66-74 